\title{
Mathematical Modeling and Stability Analysis of the Brain Tumor Glioblastoma Multiforme (GBM)
}

\author{
F. Bozkurt
}

\begin{abstract}
In this paper, a brain tumor growth that is known as Glioblastoma Multiforme (GBM) is modeled, which has two sub-population; the sensitive tumor cell and the resistant tumor cell. Within a single tumor of monoclonal origin, the sensitive cell produces another population, the resistant cell population, that has more resistance to the drug than the sensitive tumor population. In this work, the local and global stability of the positive equilibrium point of the constructed system was investigated based on specific conditions. The boundedness nature and the damped oscillation behavior of the solutions were also analyzed. The obtained stability relations depend to the growth rates of the tumor population and the drug treatment, that was considered in the discussion part of this work.
\end{abstract}

Index Terms-Difference equations, local stability, global stability, damped oscillation.

\section{INTRODUCTION}

The most severe grade of astrocytic brain tumor glioblastoma multiforme (GBM) is one of the deadliest forms of human cancer [1]. Within a single tumor of monoclonal origin, there can develop multiple sub-populations, each of which may be characterized by different growth-rates and treatment susceptibilities [2]-[5]. Mathematical approaches to tumor treatment offer a perspective that current in vivo/in vitro techniques cannot [6]-[7].

Mathematical approximation for population growth involves in some biological situations nonlinear differential equations. For an overlapping generation of a single species, a model with a differential equation is preferred. If there is a non-overlapping generation of a single species, then it is convenient to construct a model with a difference equation. For both time situations, continuous and discrete, there are some population which need the properties of both differential and difference equations, where the use of piecewise constant arguments come into question. Some works about constructing population dynamics in view of the time step can be shown in [8]-[13], [14]-[25].

In this paper, two sub-population of a GBM, sensitive cells and resistant cells, is modeled such as

$$
\left\{\begin{array}{c}
\frac{d x}{d t}=p x(t)+r_{1} x(t)\left(R_{1}-\alpha_{1} x(t)-\alpha_{2} x(\llbracket t-1 \rrbracket)\right) \\
-\gamma_{1} x(t) y(\llbracket t-1 \rrbracket)-d_{1} x(t) x(\llbracket t \rrbracket) \\
\frac{d y}{d t}=r_{2} y(t)\left(R_{2}-\beta_{1} y(t)-\beta_{2} y(\llbracket t-1 \rrbracket)\right) \\
+\gamma_{1} x(\llbracket t \rrbracket) y(t)-d_{2} y(t) y(\llbracket t \rrbracket)
\end{array}\right.
$$

Manuscript received March 3, 2014; revised May 5, 2014

F. Bozkurt is with the Erciyes University, Faculty of Education, Department of Mathematics, 38039 Kayseri, Turkey (e-mail: fbozkurt@erciyes.edu.tr). where $t \geq 0$ the parameters $\alpha_{1}, \alpha_{2}, \beta_{1}, \beta_{2}, \gamma_{1}, p, d_{1}, d_{2}, R_{1}, R_{2}$ $r_{1}$ and $r_{2}$ denote positive numbers and $\llbracket t \rrbracket$ denotes the integer part of $t \in[0, \infty) . p$ is the division rate of the sensitive cells. $R_{1}$ and $R_{2}$ are the capacities of the sensitive (including negrotic part) and resistant cell, respectively. It can be shown that $\alpha_{1}, \alpha_{2}, \beta_{1}$ and $\beta_{2}$ are parameters to construct logistic differential equations. $\gamma_{1}$ is the converting rate of sensitive cells to resistant cells. The parameters $d_{1}$ and $d_{2}$ are their dead rate caused from drugs, respectively. In Section II the local and global stability of the positive equilibrium point of system (1) and the boundedness nature of the positive solutions were investigated based on specific conditions. The damped oscillation behavior of the solutions was analyzed in Section III. Examples show the behavior of the constructed model. The discussion part in Section V will take in account the relation between the growth rate of the tumors and the drug treatment.

\section{LOCAL AND GLOBAL ASYMPTOTIC STABILITY}

In this section, the local and global stability analysis of the positive critical point of system (1) was presented.

On an interval of the form $t \in[n, n+1)$ and taking $t \rightarrow n+1$ one can write the solutions of (1) as

$$
\left\{\begin{array}{c}
x(n+1)=x(\mathrm{n}) \cdot\left\{p+r_{1} R_{1}-\alpha_{2} r_{1} x(n-1)-\gamma_{1} y(n-1)-d_{1} x(n)\right\} \\
\left(\left\{p+r_{1} R_{1}-\alpha_{2} r_{1} x(n-1)-\gamma_{1} y(n-1)-\left(\alpha_{1} r_{1}+d_{1}\right) x(n)\right\}\right. \\
\left.\exp \left(-\left\{p+r_{1} R_{1}-\alpha_{2} r_{1} x(n-1)-\gamma_{1} y(n-1)-d_{1} x(n)\right\}\right)+\alpha_{1} r_{1} x(n)\right)^{-1} \\
y(n+1)=y(n) \cdot\left\{r_{2} R_{2}-\beta_{2} r_{2} y(n-1)+\gamma_{1} x(n)-d_{2} y(n)\right\} \\
\left(\left\{r_{2} R_{2}-\beta_{2} r_{2} y(n-1)+\gamma_{1} x(n)-\left(\beta_{1} r_{2}+d_{2}\right) y(n)\right\}\right. \\
\left.\cdot \exp \left(-\left\{r_{2} R_{2}-\beta_{2} r_{2} y(n-1)+\gamma_{1} x(n)-d_{2} y(n)\right\}\right)+\beta_{1} r_{2} y(n)\right)^{-1},
\end{array}\right.
$$

where hereafter

$$
\left\{\begin{array}{c}
p+r_{1} R_{1}-\alpha_{2} r_{1} x(n-1)-\gamma_{1} y(n-1)-d_{1} x(n) \neq 0 \\
r_{2} R_{2}-\beta_{2} r_{2} y(n-1)+\gamma_{1} x(n)-d_{2} y(n) \neq 0 .
\end{array}\right.
$$

To investigate more about the behavior of (1) we continue the analysis, since (2) is a system of difference equations. Computations reveal that the positive equilibrium points of (2) is

$$
\begin{aligned}
\mu=(\bar{x}, \bar{y})= & \left(\frac{\left(p+r_{1} R_{1}\right)\left(d_{2}+\beta_{1} r_{2}+\beta_{2} r_{2}\right)-r_{2} R_{2} \gamma_{1}}{\left(d_{1}+\alpha_{2} r_{1}+\alpha_{1} r_{1}\right)\left(d_{2}+\beta_{2} r_{2}+\beta_{1} r_{2}\right)+\gamma_{1}^{2}},\right. \\
& \left.\frac{r_{2} R_{2}\left(d_{1}+\alpha_{2} r_{1}+\alpha_{1} r_{1}\right)+\left(p+r_{1} R_{1}\right) \gamma_{1}}{\left(d_{1}+\alpha_{2} r_{1}+\alpha_{1} r_{1}\right)\left(d_{2}+\beta_{2} r_{2}+\beta_{1} r_{2}\right)+\gamma_{1}^{2}}\right)
\end{aligned}
$$


where $\gamma_{1}<\frac{\left(p+r_{1} R_{1}\right)\left(d_{2}+\beta_{1} r_{2}+\beta_{2} r_{2}\right)}{r_{2} R_{2}}$. Hereafter, let

$$
\left\{\begin{array}{c}
A=p+r_{1} R_{1}-\left(\alpha_{2} r_{1}+d_{1}\right) \bar{x}-\gamma_{1} \bar{y}>0 \\
B=r_{2} R_{2}-\left(\beta_{2} r_{2}+d_{2}\right) \bar{y}+\gamma_{1} \bar{x}>0 .
\end{array}\right.
$$

Linearizing (2) about $\mu$, we obtain

$$
\left\{\begin{array}{c}
u_{1}=\frac{\left(d_{1}+\alpha_{1} r_{1}\right) \exp (-A)-d_{1}}{\alpha_{1} r_{1}} \\
u_{2}=\frac{\alpha_{2}(\exp (-A)-1)}{\alpha_{1}} \\
u_{4}=\frac{\gamma_{1}(\exp (-A)-1)}{\alpha_{1} r_{1}}
\end{array},\left\{\begin{array}{c}
v_{1}=\frac{\gamma_{1}(1-\exp (-B))}{\beta_{1} r_{2}} \\
v_{3}=\frac{\left(\beta_{1} r_{2}+d_{2}\right) \exp (-B)-d_{2}}{\beta_{1} r_{2}} \\
v_{4}=\frac{\beta_{2}(\exp (-B)-1)}{\beta_{1}}
\end{array}\right.\right.
$$

where

$$
\begin{aligned}
& \lambda^{4}-\left(u_{1}+v_{3}\right) \lambda^{3}+\left(u_{1} v_{3}-v_{4}-u_{2}\right) \lambda^{2} \\
& +\left(u_{1} v_{4}+u_{2} v_{3}-u_{4} v_{1}\right) \lambda+u_{2} v_{4}=0
\end{aligned}
$$

is the characteristic equation of (2).

Theorem 2.1. Let $(\bar{x}, \bar{y})$ be the equilibrium point of (2) and assume that the conditions $\frac{\alpha_{2} \beta_{2} d_{1}-\alpha_{1} \beta_{1}}{\alpha_{2}^{2} \beta_{2}+\alpha_{1}^{2} \beta_{1}}<r_{1}<\frac{d_{1}}{\alpha_{2}+\alpha_{1}}$, $r_{2}>\frac{d_{2}}{\beta_{2}+\beta_{1}}$ and $\frac{\alpha_{1} \beta_{1}}{\alpha_{2} \beta_{2}}<d_{1}<\frac{\left(\alpha_{1}+\alpha_{2}\right) \beta_{1}}{\alpha_{2} \beta_{2}-\alpha_{1} \beta_{1}}$ hold, where $\alpha_{2}>\alpha_{1}$ and $\frac{\alpha_{2}}{\alpha_{1}}>\frac{\beta_{1}}{\beta_{2}}$. If

$$
\begin{aligned}
& \ln \left(\frac{\alpha_{2} \beta_{2} r_{1} r_{2}+\alpha_{1} \beta_{2} r_{1} r_{2}+\beta_{1} r_{2} d_{1}+d_{1} d_{2}}{\alpha_{1} \beta_{1} r_{1} r_{2}+\alpha_{1} \beta_{2} r_{1} r_{2}+\alpha_{2} \beta_{1} r_{1} r_{2}+\alpha_{2} \beta_{2} r_{1} r_{2}+d_{1} d_{2}}\right)<B \\
& <\ln \left(\frac{\alpha_{1} \beta_{1} r_{1} r_{2}+\alpha_{2} \beta_{2} r_{1} r_{2}+\alpha_{1} r_{1} d_{2}+\beta_{1} r_{2} \mathrm{~d}_{1}+\mathrm{d}_{1} \mathrm{~d}_{2}}{\alpha_{2} \beta_{2} \mathrm{r}_{1} \mathrm{r}_{2}+\alpha_{2} \beta_{1} \mathrm{r}_{1} \mathrm{r}_{2}+\alpha_{1} \mathrm{r}_{1} \mathrm{~d}_{2}+\mathrm{d}_{1} \mathrm{~d}_{2}}\right),
\end{aligned}
$$

Then the positive equilibrium point of (2) is locally asymptotically stable.

Proof. By the Jury Condition (or Schur-Cohn criteria, see [26]) we get that the positive equilibrium point of system (2) is locally asymptotically stable if

$$
\begin{gathered}
\text { (a) } p(1)=1-\left(u_{1}+v_{3}\right)+\left(u_{1} v_{3}-v_{4}-u_{2}\right)+ \\
\left(u_{1} v_{4}+u_{2} v_{3}-u_{4} v_{1}\right)+u_{2} v_{4}>0 \\
\text { (b) }(-1)^{4} p(-1)=1+\left(u_{1}+v_{3}\right)+\left(u_{1} v_{3}-v_{4}-u_{2}\right) \\
-\left(u_{1} v_{4}+u_{2} v_{3}-u_{4} v_{1}\right)+u_{2} v_{4}>0
\end{gathered}
$$

$$
\text { (c) }\left|u_{2} v_{4}\right|<1
$$

Hold. From (a) and (b), we can write

$$
1+u_{1} v_{3}+u_{2} v_{4}>v_{4}+u_{2}
$$

Using (6) in (9), we have

$$
\begin{aligned}
& 1+\left(\frac{\left(d_{1}+\alpha_{1} r_{1}\right) e^{-\mathrm{A}}-d_{1}}{\alpha_{1} \mathrm{r}_{1}}\right)\left(\frac{\left(\beta_{1} r_{2}+d_{2}\right) e^{-B}-d_{2}}{\beta_{1} \mathrm{r}_{2}}\right) \\
& +\left(\frac{\alpha_{2}\left(\mathrm{e}^{-\mathrm{A}}-1\right)}{\alpha_{1}}\right)\left(\frac{\beta_{2}\left(e^{-B}-1\right)}{\beta_{1}}\right)>\frac{\beta_{2}\left(e^{-B}-1\right)}{\beta_{1}}+\frac{\alpha_{2}\left(e^{-A}-1\right)}{\alpha_{1}} .
\end{aligned}
$$

By ordering and computing (10), we obtain

$$
\begin{aligned}
& \ln \left(\frac{\alpha_{2} \beta_{2} r_{1} r_{2}+\alpha_{1} \beta_{2} r_{1} r_{2}+\beta_{1} r_{2} d_{1}+d_{1} d_{2}}{\alpha_{1} \beta_{1} r_{1} r_{2}+\alpha_{1} \beta_{2} r_{1} r_{2}+\alpha_{2} \beta_{1} r_{1} r_{2}+\alpha_{2} \beta_{2} r_{1} r_{2}+d_{1} d_{2}}\right)<B \\
& \quad<\ln \left(\frac{\alpha_{1} \beta_{1} r_{1} r_{2}+\alpha_{2} \beta_{2} r_{1} r_{2}+\alpha_{1} r_{1} d_{2}+\beta_{1} r_{2} d_{1}+d_{1} d_{2}}{\alpha_{2} \beta_{2} r_{1} r_{2}+\alpha_{2} \beta_{1} r_{1} r_{2}+\alpha_{1} r_{1} d_{2}+d_{1} d_{2}}\right),
\end{aligned}
$$

where $r_{1}<\frac{d_{1}}{\alpha_{2}+\alpha_{1}}<\frac{d_{1}}{\alpha_{2}-\alpha_{1}}, r_{2}>\frac{d_{2}}{\beta_{2}+\beta_{1}}$ and $\alpha_{2}>\alpha_{1}$.
Considering $(c)$, we have

$$
\frac{\alpha_{2}\left(1-e^{-A}\right)}{\alpha_{1}} \cdot \frac{\beta_{2}\left(1-e^{-B}\right)}{\beta_{1}}<1
$$

where $A>0$ and $B>0$. In view of (11), we can write

$$
\begin{gathered}
\frac{\beta_{1} r_{2}\left(d_{1}-\left(\alpha_{2}+\alpha_{1}\right) r_{1}\right)}{\alpha_{2} \beta_{2} r_{1} r_{2}+\alpha_{1} \beta_{2} r_{1} r_{2}+\beta_{1} r_{2} d_{1}+d_{1} d_{2}}<1-e^{-B} \\
<\frac{\beta_{1} r_{2}\left(d_{1}-\left(\alpha_{2}-\alpha_{1}\right) r_{1}\right)}{\alpha_{1} \beta_{1} r_{1} r_{2}+\alpha_{2} \beta_{2} r_{1} r_{2}+\alpha_{1} r_{1} d_{2}+\beta_{1} r_{2} d_{1}+d_{1} d_{2}} .
\end{gathered}
$$

Using (13) in (12), we obtain

$$
\begin{gathered}
\frac{\alpha_{2} \beta_{2}\left(1-e^{-A}\right)\left(1-e^{-B}\right)}{\alpha_{1} \beta_{1}} \\
<\frac{\alpha_{2} \beta_{2} r_{2}\left(d_{1}-\left(\alpha_{2}-\alpha_{1}\right) r_{1}\right)\left(1-e^{-A}\right)}{\alpha_{1}\left(\alpha_{1} \beta_{1} r_{1} r_{2}+\alpha_{2} \beta_{2} r_{1} r_{2}+\alpha_{1} r_{1} d_{2}+\beta_{1} r_{2} d_{1}+d_{1} d_{2}\right)}<1
\end{gathered}
$$

which lead us to the inequality

$$
\begin{aligned}
& \frac{\alpha_{2} \beta_{2} r_{2}\left(d_{1}-\left(\alpha_{2}-\alpha_{1}\right) r_{1}\right)}{\alpha_{1}\left(\alpha_{1} \beta_{1} r_{1} r_{2}+\alpha_{2} \beta_{2} r_{1} r_{2}+\alpha_{1} r_{1} d_{2}+\beta_{1} r_{2} d_{1}+d_{1} d_{2}\right)}-1 \\
& <\frac{\alpha_{2} \beta_{2} r_{2}\left(\mathrm{~d}_{1}-\left(\alpha_{2}-\alpha_{1}\right) \mathrm{r}_{1}\right)}{\alpha_{1}\left(\alpha_{1} \beta_{1} r_{1} r_{2}+\alpha_{2} \beta_{2} r_{1} r_{2}+\alpha_{1} r_{1} d_{2}+\beta_{1} r_{2} d_{1}+d_{1} d_{2}\right)} e^{-A} .
\end{aligned}
$$

This is simplify such as

$$
\begin{gathered}
\alpha_{2} \beta_{2} r_{2}\left(d_{1}-\left(\alpha_{2}-\alpha_{1}\right) r_{1}\right)-\alpha_{1}\left(\begin{array}{c}
\alpha_{1} \beta_{1} r_{1} r_{2}+\alpha_{2} \beta_{2} r_{1} r_{2}+ \\
\alpha_{1} \mathrm{r}_{1} \mathrm{~d}_{2}+\beta_{1} \mathrm{r}_{2} \mathrm{~d}_{1}+\mathrm{d}_{1} \mathrm{~d}_{2}
\end{array}\right)< \\
\alpha_{2} \beta_{2} r_{2}\left(d_{1}-\left(\alpha_{2}-\alpha_{1}\right) r_{1}\right) e^{-A} .
\end{gathered}
$$

Since

$$
\frac{\alpha_{1} \beta_{1}}{\alpha_{2} \beta_{2}}<d_{1}<\frac{\left(\alpha_{1}+\alpha_{2}\right) \beta_{1}}{\alpha_{2} \beta_{2}-\alpha_{1} \beta_{1}}
$$

And $\frac{\alpha_{2}}{\alpha_{1}}>\frac{\beta_{1}}{\beta_{2}}$

where

$$
\frac{\alpha_{2} \beta_{2} d_{1}-\alpha_{1} \beta_{1}}{\alpha_{2}^{2} \beta_{2}+\alpha_{1}^{2} \beta_{1}}<r_{1}<\frac{d_{1}}{\alpha_{2}+\alpha_{1}}
$$

The left side of the inequality (15) will be negative. This completes the proof.

Theorem 2.2. Let $\{(x(n), y(n))\}_{n=0}^{\infty}$ be a positive solution of (2). Assume that for $n=0,1, \ldots$ the conditions

$$
\left\{\begin{array}{c}
\alpha_{1} r_{1} x(n)<p+r_{1} R_{1}-\alpha_{2} r_{1} x(n-1) \\
-\gamma_{1} y(n-1)-d_{1} x(n) \\
\beta_{1} r_{2} y(n)<r_{2} R_{2}-\beta_{2} r_{2} y(n-1) \\
+\gamma_{1} x(n)-d_{2} y(n)
\end{array}\right.
$$

Holds. The following statements are true.

All positive solutions of (2) are in the interval

And

$$
x(n) \in\left(0, \frac{p+r_{1} R_{1}}{\alpha_{1} r_{1}}\right)
$$

$$
y(n) \in\left(0, \frac{\alpha_{1} R_{2} r_{1} r_{2}+\gamma_{1}\left(p+r_{1} R_{1}\right)}{\alpha_{1} \beta_{1} r_{1} r_{2}}\right) .
$$

The solution of system (2) increase monotonic.

Theorem 2.3. Let system (2) be 


$$
\begin{gathered}
F(x(n), y(n), x(n-1), y(n-1))= \\
\{x(n+1)=f(x(n), y(n), x(n-1), y(n-1)) \\
y(n+1)=g(x(n), y(n), x(n-1), y(n-1))
\end{gathered}
$$

where the first order partial derivatives of the functions $f$ and $\mathrm{g}$ regard to $\mathrm{x}$ and $\mathrm{y}$ are continuous in $I \subset R^{+}$and $f, g: V \subset$ $\left(\mathbb{R}^{+}\right)^{4} \rightarrow I \subset R^{+}$. Furthermore assume that

$$
\begin{gathered}
\alpha_{1} r_{1} x(n)<p+r_{1} R_{1}-\alpha_{2} r_{1} x(n-1) \\
-\gamma_{1} y(n-1)-d_{1} x(n)
\end{gathered}
$$

And

$$
\begin{gathered}
\beta_{1} r_{2} y(n)<r_{2} R_{2}-\beta_{2} r_{2} y(n-1)+\gamma_{1} x(n) \\
-d_{2} y(n),
\end{gathered}
$$

That $2 \alpha_{1}>\alpha_{2}>\alpha_{1}, 2 \beta_{1}>\beta_{2}>\beta_{1}$ and $\gamma_{1}<d_{1}$. If

$$
\frac{\alpha_{1} r_{1} \times(n)}{1-\alpha_{2} r_{1} x(n)}<A<\ln \left(\frac{A-\alpha_{1} r_{1} x(n)}{\alpha_{2} r_{1} A x(n)}\right)
$$

And

$$
\frac{\beta_{1} r_{2} y(n)}{1-\beta_{2} r_{2} y(n)}<B<\ln \left(\frac{B-\beta_{1} r_{2} y(n)}{\beta_{2} r_{2} B y(n)}\right)
$$

where $r_{1}>\frac{d_{1}}{\alpha_{1}}, r_{2}>\frac{d_{2}}{\beta_{1}}, x(n)<\frac{1}{\alpha_{2} r_{1}}$ and $y(n)<\frac{1}{\beta_{1} r_{2}}$, then (17) has no 2-cycle in $I$.

Proof. For some

$$
\left\{\begin{array}{l}
s(2)=(x(1), y(1), x(0), y(0)) \\
s(3)=(x(2), y(2), x(1), y(1)) \\
r(2)=(x(1), y(1), x(0), y(0)) \\
r(3)=(x(2), y(2), x(1), y(1))
\end{array}\right.
$$

$A_{2}$-cycle will be hold the condition

$$
\left\{\begin{array}{l}
s(2)=f(s(3))=f(f(x(1), y(1), x(0), y(0))) \\
r(2)=g(r(3))=g(g(x(1), y(1), x(0), y(0))) .
\end{array}\right.
$$

In this case, we must have $\int_{s(2)}^{s(3)}\left(1+\frac{\partial f}{\partial x}\right) d x \neq 0$, $\int_{s(2)}^{s(3)}\left(1+\frac{\partial f}{\partial y}\right) d y \neq 0 \quad \int_{r(2)}^{r(3)}\left(1+\frac{\partial g}{\partial x}\right) d x \neq 0 \quad$ and $\int_{r(2)}^{r(3)}(1+$ $\left.\frac{\partial g}{\partial y}\right) d y \neq 0$. This lead us to the following cases:

The partial derivative of $f(x(n), y(n), x(n-1), y(n-$ 1)) regard to $x(n), x(n-1), y(n)$ and $y(n-1)$ will give the following results:

For the partial derivative of function $\mathrm{f}$ regard to $x(n)$, we get

$$
\begin{gathered}
1+\frac{\partial f}{\partial x(n)}=\frac{1}{\left(\left(A-\alpha_{1} r_{1} x(n)\right) e^{-A}+\alpha_{1} r_{1} x(n)\right)^{2}} \\
\times\left\{\left(A-\alpha_{1} r_{1} x(n)\right) e^{-A} x(n)\left(2 \alpha_{1} r_{1}-d_{1}\right)\right. \\
+\left(A-\alpha_{1} r_{1} x(n)\right) e^{-A}\left(\left(A-\alpha_{1} r_{1} x(n)\right) e^{-A}-d_{1} A x(n)\right) \\
+\alpha_{1} r_{1}(x(n))^{2}\left(\alpha_{1} r_{1}-d_{1}\right)+\left(A-\alpha_{1} r_{1} x(n)\right) A \mathrm{e}^{-A} \\
\left.+\left(\alpha_{1} r_{1}+d_{1}\right) A x(n) e^{-A}\right\} .
\end{gathered}
$$

Since $r_{1}>\frac{d_{1}}{\alpha_{1}}$, if

$$
\left(A-\alpha_{1} r_{1} x(n)\right) e^{-A}-d_{1} A x(n)>0,
$$

Then $1+\frac{\partial f}{\partial x(n)}>0$. The inequality (25) leads to the condition

$$
\frac{\alpha_{1} r_{1} x(n)}{1-d_{1} x(n)}<A<\ln \left(\frac{A-\alpha_{1} r_{1} x(n)}{d_{1} A x(n)}\right)
$$

where $x(n)<\frac{1}{d_{1}}$. Showing the partial derivative of function $f$ regard to $x(n-1)$, give that $1+\frac{\partial f}{\partial x(n-1)}>0$, if

$$
\frac{\alpha_{1} r_{1} x(n)\left(\alpha_{2}-\alpha_{1}\right)}{\alpha_{2}}<A<\ln \left(\frac{\alpha_{2} \mathrm{~A}}{\alpha_{1} r_{1} x(n)\left(\alpha_{2}-\alpha_{1}\right)}\right)
$$

And

$$
\frac{\alpha_{1} r_{1} x(n)}{1-\alpha_{2} r_{1} x(n)}<A<\ln \left(\frac{A-\alpha_{1} r_{1} x(n)}{\alpha_{2} r_{1} A x(n)}\right)
$$

where $2 \alpha_{1}>\alpha_{2}>\alpha_{1}$ and $\mathrm{x}(\mathrm{n})<\frac{1}{\alpha_{2} \mathrm{r}_{1}}$. Considering both (27) and (28), since $\alpha_{2}>\alpha_{1}$, we get

$$
\begin{aligned}
& \frac{\alpha_{1} r_{1} x(n)\left(\alpha_{2}-\alpha_{1}\right)}{\alpha_{2}}<\frac{\alpha_{1} r_{1} x(n)}{1-\alpha_{2} r_{1} x(n)}<A \\
< & \ln \left(\frac{A-\alpha_{1} r_{1} x(n)}{\alpha_{2} r_{1} A x(n)}\right)<\ln \left(\frac{\alpha_{2} A}{\alpha_{1} r_{1} x(n)\left(\alpha_{2}-\alpha_{1}\right)}\right) .
\end{aligned}
$$

The partial derivative of function $\mathrm{f}$ regard to $y(n)$ leads to $1+\frac{\partial f}{\partial y(n)}=1>0$. At last, we can write

$$
\begin{gathered}
1+\frac{\partial f}{\partial y(n-1)}=\frac{1}{\left(\left(A-\alpha_{1} r_{1} x(n)\right) e^{-A}+\alpha_{1} r_{1} x(n)\right)^{2}} \\
\times\left\{\left(A-\alpha_{1} r_{1} x(n)\right) e^{-A}\left(\left(A-\alpha_{1} r_{1} x(n)\right) e^{-A}-\gamma_{1} A x(n)\right)\right. \\
+\alpha_{1} r_{1}(x(n))^{2}\left(\alpha_{1} r_{1}-\gamma_{1}\right)+\left(A-\alpha_{1} r_{1} x(n)\right) \\
\left.e^{-A} x(n)\left(2 \alpha_{1} r_{1}-\gamma_{1}\right)+\gamma_{1} A x(n) e^{-A}\right\}
\end{gathered}
$$

Since $x(n)<\frac{1}{\gamma_{1}}$ and $r_{1}>\frac{\gamma_{1}}{2 \alpha_{1}}$, if

$$
\frac{\alpha_{1} r_{1} x(n)}{1-\gamma_{1} x(n)}<A<\ln \left(\frac{A-\alpha_{1} r_{1} x(n)}{\gamma_{1} A x(n)}\right)
$$

Then we get $1+\frac{\partial f}{\partial y(n-1)}>0$. Taking in view (26), (29) and (31), we obtain

$$
\begin{aligned}
\frac{\alpha_{1} r_{1} x(n)}{1-\gamma_{1} x(n)}<\frac{\alpha_{1} r_{1} x(n)}{1-d_{1} x(n)}<\frac{\alpha_{1} r_{1} x(n)}{1-\alpha_{2} r_{1} x(n)}<A \\
<\ln \left(\frac{A-\alpha_{1} r_{1} x(n)}{\alpha_{2} r_{1} A x(n)}\right)<\ln \left(\frac{A-\alpha_{1} r_{1} x(n)}{d_{1} A x(n)}\right)<\ln \left(\frac{A-\alpha_{1} r_{1} x(n)}{\gamma_{1} A x(n)}\right)
\end{aligned}
$$

where $\gamma_{1}<d_{1}, r_{1}>\frac{d_{1}}{\alpha_{2}}>\frac{\gamma_{1}}{\alpha_{2}}$ and $x(n)<\frac{1}{\alpha_{2} r_{1}}<\frac{1}{d_{1}}<\frac{1}{\gamma_{1}}$.

The partial Derivative of $g(x(n), y(n), x(n-1), y(n-$ 1)) regard to $x(n), x(n-1), y(n)$ and $y(n-1)$ will give the following results: For

$$
1+\frac{\partial g}{\partial y(n)}=\beta_{1} r_{2}(y(n))^{2}\left(\beta_{1} r_{2}-d_{2}\right)+\left(B-\beta_{1} r_{2} y(n)\right)
$$




$$
\begin{gathered}
e^{-B} y(n)\left(2 \beta_{1} r_{2}-d_{2}\right)+\left(B-\beta_{1} r_{2} y(n)\right) e^{-B} \\
\left(\left(B-\beta_{1} r_{2} y(n)\right) e^{-B}-d_{2} B y(n)\right)+\left(\beta_{1} r_{2}+d_{2}\right) e^{-B} B y(n) \\
+B\left(B-\beta_{1} r_{2} y(n)\right) e^{-B}
\end{gathered}
$$

if

$$
\frac{\beta_{1} r_{2} y(n)}{1-d_{2} y(n)}<B<\ln \left(\frac{B-\beta_{1} r_{2} y(n)}{d_{2} B y(n)}\right),
$$

where $r_{2}>\frac{d_{2}}{\beta_{1}}$ and $y(n)<\frac{1}{d_{2}}$, then one can obtain

Considering

$$
1+\frac{\partial g}{\partial y(n)}>0
$$

$$
\begin{aligned}
& 1+\frac{\partial g}{\partial y(n-1)}=\left(B-\beta_{1} r_{2} y(n)\right) e^{-B} \\
& \left(\left(B-\beta_{1} r_{2} y(n)\right) e^{-B}-\beta_{2} r_{2} B y(n)\right) \\
+ & \beta_{1} r_{2}{ }^{2} y(n)^{2}\left(\beta_{1}-\beta_{2}\right)+\beta_{2} r_{2} e^{-B} B y(n) \\
+ & \left(B-\beta_{1} r_{2} y(n)\right) e^{-B} y(n) r_{2}\left(2 \beta_{1}-\beta_{2}\right),
\end{aligned}
$$

We obtain that since $2 \beta_{1}>\beta_{2}>\beta_{1}$ and $y(n)<\frac{1}{\beta_{1} r_{2}}$, if

$$
\frac{\left(\beta_{2}-\beta_{1}\right) \beta_{1} \mathrm{r}_{2} \mathrm{y}(\mathrm{n})}{\beta_{2}}<B<\ln \left(\frac{\beta_{2} \mathrm{~B}}{\left(\beta_{2}-\beta_{1}\right) \beta_{1} \mathrm{r}_{2} \mathrm{y}(\mathrm{n})}\right)
$$

And

$$
\frac{\beta_{1} r_{2} y(n)}{1-\beta_{2} r_{2} y(n)}<B<\ln \left(\frac{B-\beta_{1} r_{2} y(n)}{\beta_{2} r_{2} B y(n)}\right)
$$

Then $1+\frac{\partial g}{\partial y(n-1)}>0$. Considering both (34) and (35), since $\beta_{2}>\beta_{1}$, we obtain

$$
\begin{aligned}
& \frac{\left(\beta_{2}-\beta_{1}\right) \beta_{1} r_{2} y(n)}{\beta_{2}}<\frac{\beta_{1} r_{2} y(n)}{1-\beta_{2} r_{2} y(n)}<B \\
& <\ln \left(\frac{B-\beta_{1} r_{2} y(n)}{\beta_{2} r_{2} B y(n)}\right)<\ln \left(\frac{\beta_{2} B}{\left(\beta_{2}-\beta_{1}\right) \beta_{1} r_{2} y(n)}\right) .
\end{aligned}
$$

Showing similarly $1+\frac{\partial g}{\partial x(n)}$ and $1+\frac{\partial g}{\partial x(n-1)}$, one can see that both are always positive. Considering at last (33) and (36), we get

$$
\begin{gathered}
\frac{\beta_{1} r_{2} y(n)}{1-d_{2} y(n)}<\frac{\beta_{1} r_{2} y(n)}{1-\beta_{2} r_{2} y(n)}<B<\ln \left(\frac{B-\beta_{1} r_{2} y(n)}{\beta_{2} r_{2} B y(n)}\right)< \\
\ln \left(\frac{B-\beta_{1} r_{2} y(n)}{d_{2} B y(n)}\right),
\end{gathered}
$$

where $r_{2}>\frac{d_{2}}{\beta_{1}}>\frac{d_{2}}{\beta_{2}}$ and $y(n)<\frac{1}{\beta_{1} r_{2}}<\frac{1}{d_{2}}$.

Some additional assumptions of (18) are needed to verify global asymptotic stability for the positive equilibrium point $(\bar{x}, \bar{y})$.

$f_{-}$and $g$ are continuous functions.

$$
\begin{aligned}
f:\left(0, \frac{p+r_{1} R_{1}}{\alpha_{1} r_{1}}\right) & \times\left(0, \frac{\alpha_{1} R_{2} r_{1} r_{2}+\gamma_{1}\left(p+r_{1} R_{1}\right)}{\alpha_{1} \beta_{1} r_{1} r_{2}}\right) \\
& \rightarrow\left(0, \frac{p+r_{1} R_{1}}{\alpha_{1} r_{1}}\right) \\
g:\left(0, \frac{p+r_{1} R_{1}}{\alpha_{1} r_{1}}\right) & \times\left(0, \frac{\alpha_{1} R_{2} r_{1} r_{2}+\gamma_{1}\left(p+r_{1} R_{1}\right)}{\alpha_{1} \beta_{1} r_{1} r_{2}}\right)
\end{aligned}
$$

$$
\rightarrow\left(0, \frac{\alpha_{1} R_{2} r_{1} r_{2}+\gamma_{1}\left(p+r_{1} R_{1}\right)}{\alpha_{1} \beta_{1} r_{1} r_{2}}\right)
$$

Corollary 2.1. Let $(\bar{x}, \bar{y})$ be a positive equilibrium point of system (2). Suppose that Theorem 2.1-Theorem 2.3 hold. Then the positive equilibrium point of system (2) is globally asymptotically stable.

\section{Oscillation}

In this section is the damped oscillation behavior of the solutions of system (2) was considered.

Theorem 3.1. Let $\{(x(n), y(n))\}_{n=0}^{\infty}$ be a positive solution of system (2). Assume that

$$
\begin{gathered}
\alpha_{1} r_{1} x(2 n)<p+r_{1} R_{1}-\alpha_{2} r_{1} x(2 n-1)-\gamma_{1} y(2 n-1)- \\
d_{1} x(2 n)<\ln \left(\frac{x(2 n-1)}{x(2 n)}\right)
\end{gathered}
$$

And

$$
\begin{gathered}
\beta_{1} r_{2} y(2 n)<r_{2} R_{2}-\beta_{2} r_{2} y(2 n-1)+\gamma_{1} x(2 n)- \\
d_{2} y(2 n)<\ln \left(\frac{y(n-1)}{y(n)}\right)
\end{gathered}
$$

Hold, where $r_{1}>\frac{d_{1}}{\alpha_{2}}$ and $r_{2}>\frac{d_{2}}{\beta_{2}}$. If

$$
x(2 n)<\bar{x}<x(2 n-1)
$$

And

$$
y(2 n)<\bar{y}<y(2 n-1)
$$

Then the solution of (2) has damped oscillations.

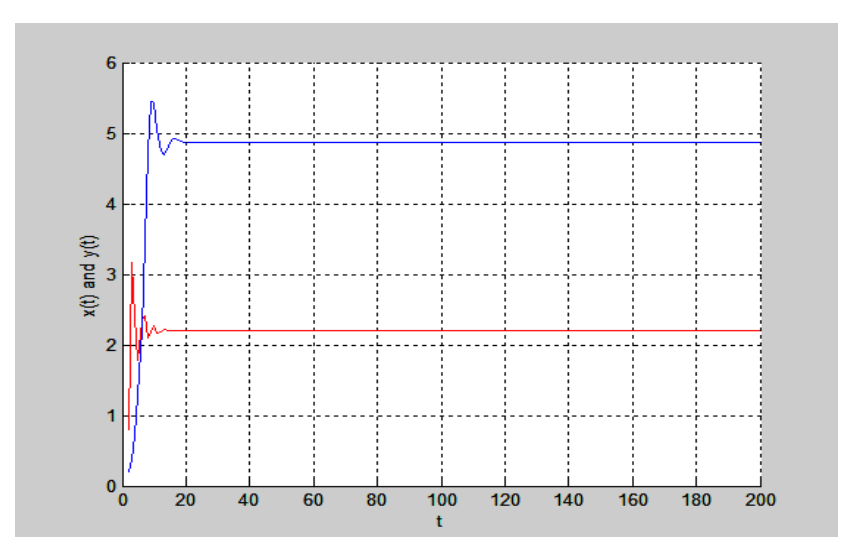

Fig. 1. Local stability of the solutions of system (2.2).

\section{EXAMPLE}

The values of the parameters of (2.2) are as selected as given in [9] and in view the obtained results. $p=0.192$ give the division rate of the sensitive cells. $R_{1}=0.42 \times 38^{2} / 3=$ 4.704 Is the carrying capacity of the negrotic and sensitive cells together, $R_{2}=0.11 \times 38^{2 / 3}=1.232$ give the carrying capacity of the resistant tumor population. The mutation rate of the sensitive cells to resistant cells is in the interval $\left[10^{-5}, 10^{-2}\right]$. In [9] the parameters for the resistant population is given as $\beta_{1}=0.05$ and $\beta_{2}=0.2$. The same parameters are used for the resistant tumor population. The 
sensitive tumor cells have population rates in the interval $[0.5,0.95]$, which lead to select $\alpha_{1}=0.51$ and $\alpha_{2}=0.555$. The causes of drug treatment to the sensitive and resistant cells are selected as $d_{1}=0.6$ and $d_{2}=0.006$, respectively. Both populations have different population rates. In this example a relation between both population are constricted such as $r=r_{1}$ and (1.05) $\times r=r_{2}$. Fig. 1 and Fig. 2 show the behavior of the sensitive and resistant tumor cells in view of the conditions of Theorem 2.1. The mutation rate is selected $\gamma_{1}=0.01$.

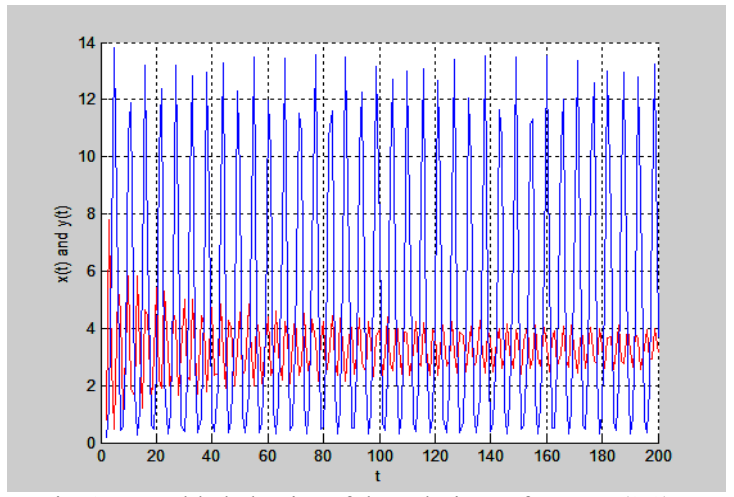

Fig. 2. Unstable behavior of the solutions of system (2.2).

To verify the conditions in Theorem 2.3 , the parameter $\beta_{1}$ is selected as $\beta_{1}=0.15$ and $r=r_{1}=0.08$. The global behavior of the tumor population can be shown in Fig. 3 .

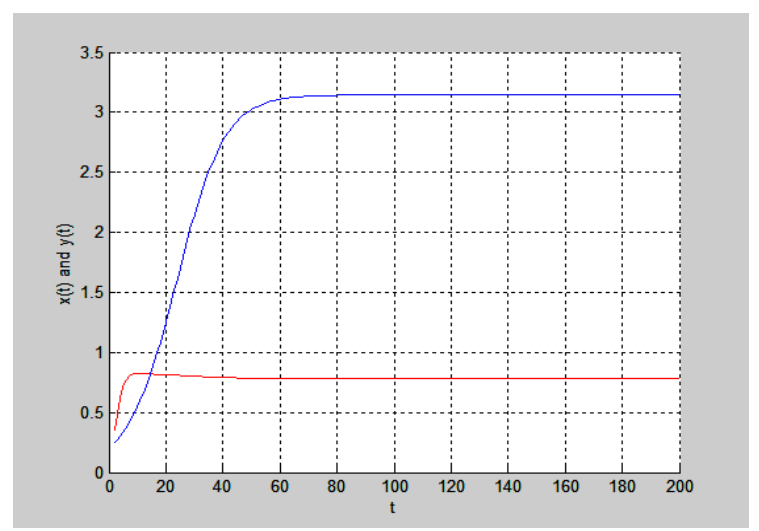

Fig. 3. Global asymptotic stability of the solution of system (2.2).

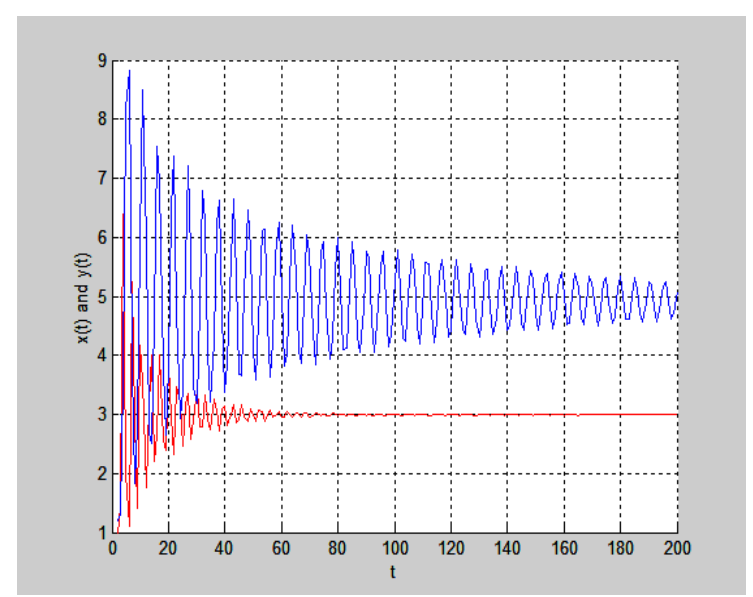

Fig. 4. Damped oscillation behavior of the solutions of system (2.2)

In view of Theorem 3.1, the growth rate is selected as $r=r_{1}=1.09$ and $\beta_{1}=0.05$. The obtained behavior can be shown in Fig. 4.
Fig. 5 and Fig. 6 show the bicurcation diagram of the tumor population where the mutation rate changed. The red and the blue graph sembolize the sensitive cells population $(x(t))$ and the resistant cells population $(y(t))$, respectively.

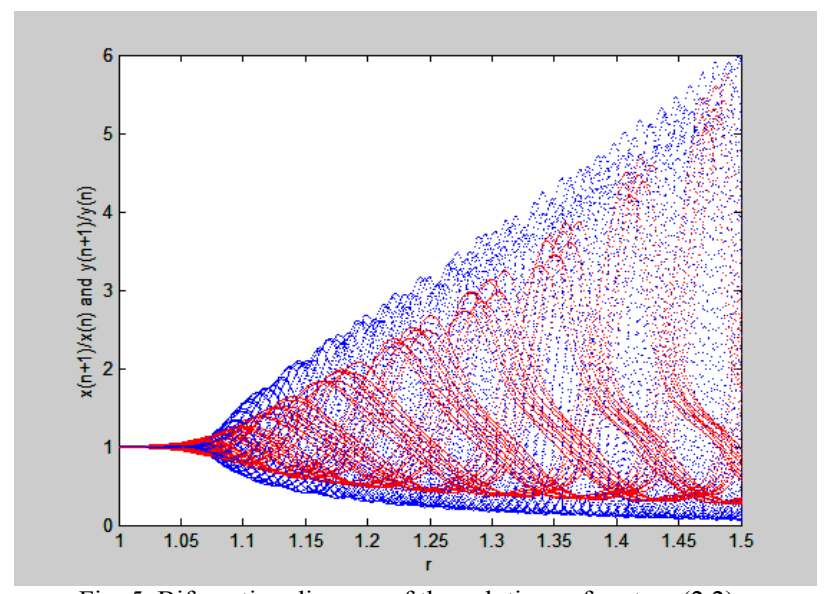

Fig. 5. Bifurcation diagram of the solutions of system (2.2).

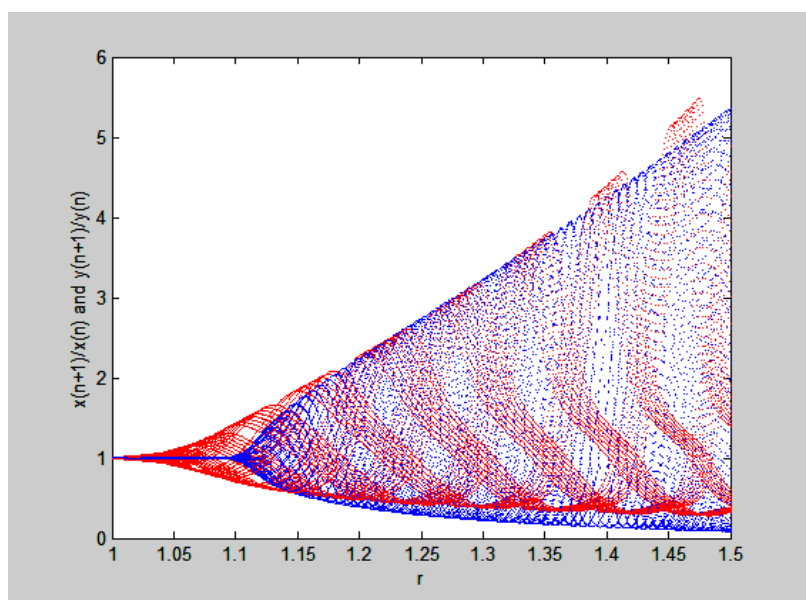

Fig. 6. Bifurcation diagram of the solutions of system (2.2).

\section{CONCLUSION}

To consider the model in (1), its solution (2) has been studied as a difference equation system. In section 2 , the local and global asymptotic stability of the positive equilibrium point of system (2) was studied in Theorem 2.1-Theorem 2.3 and Corollary 2.1. Theorem 3.1 gives information of the damped oscillation behavior of system (2). It can be shown that with the mutation rate $\gamma_{1}=0.00001$ after a certain population rate a resistant tumor population occurs over the wall of the sensitive cells. Furthermore, if the mutation rate is $\gamma_{1}=0.01$, then the resistant tumor population will cover the sensitive tumor cells. It is interesting to see that for the local and global stability a relation between the growth rates and the drug treatment is obtained.

This work shows investigations of the behavior of the tumor growth GBM. Future works will include studies about the stability of GBM in view of the density.

\section{ACKNOWLEDGMENT}

This work is supported by the Scientific Research Center at the Erciyes University with the project code FYL-2014-5122. 


\section{REFERENCES}

[1] E. C. Holland and G. Multiforme, "The terminator," Nat. Acad. of Sci., 2000, vol. 97, pp. 6242-6244,.

[2] Y. A. Yung, J. R. Shapiro, and W. R. Shapiro, "Heterogeneous chemosensitivities of subpopulations of human glioma cells in culture," Cancer Res., vol. 42, pp. 992-998, 1982.

[3] W. Paulus and J. Peiffer, "Intratumoral histologic heterogeneity of gliomas, A quantitative study," Cancer, vol. 64, pp. 442-447, 1989.

[4] R. A. Berkman et al., "Clonal composition of glioblastoma multiforme," J. of Neurosurgery, vol. 77, pp. 432-437, 1992.

[5] S. W. Coons and P. C. Johnson, "Regional heterogeneity in the DNA content of human gliomas," Cancer, vol. 72, pp. 3052-3060, 1993.

[6] A. J. Coldman and J. H. Goldie, "A mathematical model for relating the drug sensitivity of tumors to their spontaneous mutation rate," Cancer Treat. Rep., vol. 63, pp. 1727-1731, 1979.

[7] J. C. Panetta, "A mathematical model of drug resistance: Heterogeneous tumors," Math. Biosci., vol. 147, pp. 41-61, 1998.

[8] B. G. Birkhead et al., A mathematical model of the development of drug resistance to cancer chemotherapy, Europe," J. of Cancer and Clinical Oncology, vol. 23, pp. 1421-1427, 1987.

[9] J. E. Schmitz, A. R. Kansal, and S. Torquato, "A cellular automaton of brain tumor treatment and resistance," J. of Theoret. Med., vol. 4, no. 4, pp. 223-239, 2002.

[10] J.L. Gevertz and S. Toquato, "Modeling the effects of vasculature evolution on early brain tumor growth," J. of Theoret. Biol., vol. 243, pp. 517-531, 2006.

[11] F. Gurcan and F. Bozkurt, "Global stability in a population model with piecewise constant arguments," J. of Math. Anal. Appl., vol. 360, no. 1, pp. 334-342, 2009.

[12] I. Ozturk and F. Bozkurt, "Stability analysis of a population model with piecewise constant arguments," Non. Anal.: $R W A$, vol. 12, issue 3, pp. 1532-1545, 2011.

[13] I. Ozturk, F. Bozkurt, and F. Gurcan, "Stability analysis of a mathematical model in a microcosm with piecewise constant arguments," Mathematical Biosciences, vol. 240, no. 2, pp. 85-91, 2012.

[14] P. Mosayebi et al., "Tumor invasion margin on the Riemannian space of brain fibers," Medical Image Analysis, vol. 16, no. 2, pp. 361-373, 2012.

[15] C. Gh. Buzea, "Some implications of scale relativity theory in avascular stages of growth of solid tumors in the presence of an immune system response," Journal of Theoretical Biology, vol. 282, no. 1, pp. 52-64, 2011.

[16] F. Bozkurt, "Stability analysis of a logistic differential equation," International Journal of Mathematics and Computation, vol. 21, no. 4, pp. 1-14, 2013.

[17] F. Bozkurt, "Stability analysis of a nonlinear difference equation with delay," International Journal of Modern Nonlinear Theory and Application, vol. 2, no. 1, pp. 1-6, 2013.

[18] F. Bozkurt, "A mathematical model of the brain tumor Gliobalstoma Multiforme (GBM) and the Immune System (IS) interaction,"
International Journal of Mathematics and Computation, vol. 22, no. 1, pp. 47-56, 2014.

[19] F. Bozkurt, "Modeling a tumor growth with piecewise constant arguments," Discrete Dynamics in Nature and Society, vol. 2013, pp. $1-8,2013$.

[20] F. Bozkurt, "Hopf bifurcation and stability analysis for a delayed logistic equation," International Journal of Modeling and Optimization, vol. 3, pp. 288-292, 2013.

[21] F. Bozkurt, "Stability analysis of a fractional-order differential equation system of a GBM-IS interaction depending on the density," Applied Mathematics and Information Science, vol. 8, no. 3, pp. 1-8, 2014.

[22] F. Bozkurt and I. Ozturk, "A population model of two-strains tumors with piecewise constant arguments," Kuwait Journal of Science, vol. 1, pp. 1-11, 2015.

[23] E. I. Kulich et al., "Phase transition in tumor growth: I avascular development," Physica A: Statistical Mechanics and its Applications, vol. 392, no. 24, pp. 6616-6623, 2013.

[24] N. C. Atuegwu et al., "Parameterizing the logistic model of tumor growth by dw-mri and dce-mri data to predict treatment response and changes in breast cancer cellularity during neoadjuvant chemotherapy," Translational Oncology, vol. 6, no. 3, pp. 256-264, 2013.

[25] M. Bodnar et al., "Logistic type equations with discrete delay and quasi-periodic suppression rate," Applied Mathematics Letters, vol. 26, no. 6, pp. 607-611, 2013.

[26] L. J. S. Allen, An Introduction to Mathematical Biology, NJ: Pearson Prentice Hall, 2007.

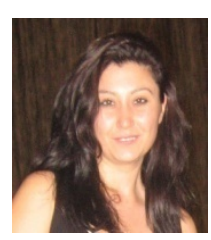

Fatma Bozkurt was born in Germany in 1979. Since 1994 she lives in Turkey.

She studied mathematics at the Erciyes University (1998-2002), and she has two MSci, which one is about applied mathematics ("The periodic character of the solutions of some difference equation", Erciyes University, Turkey, 2002-2005) and the other about mathematics education (Hacettepe University, Turkey, 2003-2005). Her PhD thesis is about the population dynamics of single species ("Stability Analysis for Differential and Difference Equations in Population Models, Erciyes University, Turkey, 2006-2010).

She worked from 2005 until 2010 as a research assistant at the Erciyes University and got the assis. prof. Dr. title in 2010. Since 2010 she is working as an assis. prof. Dr. at the Erciyes University in Kayseri (Turkey). Her research fields are about the dynamics of species and especially about modeling and analyzing the brain tumor GBM.

She is the head of some projects about modeling GBM tumor and the epidemic disease HIV/AIDS (FBA-12-4137, FBY-12-3993) and is researcher at the project FBA-11-3597. 